\title{
A Self-Calibration Scheme for TDD Massive MIMO with Imperfect Channel Estimation
}

\author{
De Mi*, Lei Zhang ${ }^{\dagger}$, Mehrdad Dianati ${ }^{\ddagger *}$, Sami Muhaidat ${ }^{\S *}$ and Pei Xiao* \\ *Institute for Communication Systems, University of Surrey, United Kingdom \\ ${ }^{\dagger}$ School of Engineering, University of Glasgow, United Kingdom \\ $\ddagger$ Warwick Manufacturing Group, University of Warwick, United Kingdom

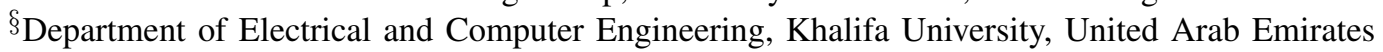 \\ Email:d.mi@surrey.ac.uk, lei.zhang@glasgow.ac.uk,m.dianati@warwick.ac.uk, \\ muhaidat@ieee.org,p.xiao@surrey.ac.uk
}

\begin{abstract}
Exploiting channel reciprocity, time-divisionduplexing (TDD) operated massive multiple-input multipleoutput (MIMO) systems are able to acquire the channel state information with a reasonable overhead of channel estimation. However, in practical scenarios, the imperfections in channel reciprocity can significantly degrade the system performance. In this work, we propose a novel self calibration scheme for the maximum ratio transmission in TDD multi-user massive MIMO systems to compensate for the imperfect channel reciprocity, with considerations of imperfect channel estimation. The proposed scheme shows the greater robustness to a compound effect of channel reciprocity error and channel estimation error, compared with the traditional self calibration scheme that is widely used in massive MIMO systems.
\end{abstract}

\section{INTRODUCTION}

Employing a large number of antennas at base stations (BS), massive (or large scale) MIMO (multiple-input multipleoutput) systems enable the service for multiple user terminals (UTs) on the same time-frequency resource, which significantly enhances the system throughput compared to the current wireless transmission techniques. Hence, the massive MIMO system has recently attracted tremendous attention and identified as a promising candidate technique for next generation wireless communications [1], [2]. By exploiting channel reciprocity in time-division duplexing (TDD) operation, the BS can estimate the downlink (DL) channel by using the uplink (UL) pilots from the UTs [3]. Hence, the overhead of the pilot transmission is proportional to the number of UTs antennas, which is typically much less than the number of BS antennas in massive MIMO systems. Therefore, TDD operation has been widely considered for the large scale antenna systems, where, in most prior studies [4], [5], the perfect channel reciprocity is considered by assuming that the time delay from the UL to the DL is within the coherence time of the channel. However, in practice, the TDD channel reciprocity is also conditioned on independent radio-frequency (RF) chains that connected to each antenna [1]. The behaviour of the RF chains is likely to be random [6], thus introducing amplitude and phase mismatches between the UL and the DL [7]. It is in turn expected that the

We would like to acknowledge the support of the University of Surrey 5GIC (www.surrey.ac.uk/5gic) members for this work. perfect channel reciprocity may not be achieved even within the coherent time, and the imperfect channel reciprocity can lead to a significant system performance degradation due to the mismatches of the realistic RF chains in TDD massive MIMO systems. Such performance degradation of linear precoding schemes has been thoroughly investigated in our prior work [8], with considerations of the imperfect channel estimation. Our analytical and simulated results in [8] show that both maximum ratio transmission (MRT) and zero-forcing (ZF) are severely affected by the compound effect of the reciprocity and estimation errors. It is therefore required to perform a suitable calibration to compensate for the imperfection of channel reciprocity.

In general, for the precoded TDD operated system, calibration schemes contain two stages which are the estimation of transmit (Tx) and receive ( $\mathrm{Rx}$ ) RF frontends' responses (or equivalently calibration coefficients), and the calculation of the calibration matrix to calibrate the precoders. To estimate the calibration coefficients, a so-called self-calibration scheme is widely considered in massive MIMO systems, since it can be implemented at the BS side only and without requiring pilots exchange between UTs and the BS during the calibration [9]. Different methods to realise self-calibration in massive MIMO systems have been provided [10], [11]. In [10], the effect of antenna coupling at the BS is utilised to measure the calibration coefficients. The method in [10] is sensitive to the scatterings near the BS antennas. A method presented in the practical study [11] contains an additional RF transceiver as a reference to exchange calibration pilots with other BS antennas' transceivers. This method is very sensitive to the placement of the reference transceiver. Studies such as [12] show that additional calibration circuits can be equipped at the BS to obtain reliable estimates of the calibration coefficients. The self-calibration scheme in [11], [12] is also known as relative calibration, i.e., relatively calibrate BS antennas based on the ratio of the $\mathrm{Tx} R F$ response to the $\mathrm{Rx} R F$ response. This has been widely considered in the context of the massive MIMO system [13], [14].

We indicated in [8] that a suitable calibration scheme for the practical scenario is required to compensate for the performance degradation caused by the compound effect of 


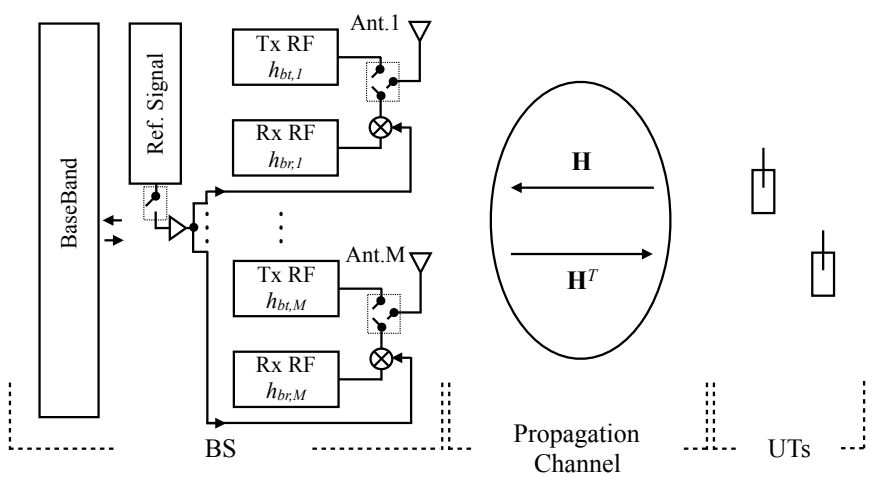

Fig. 1. A TDD MU massive MIMO System with calibration circuits.

the reciprocity and channel estimation errors. However, there have been no calibration schemes, including the widely-used relative calibration, taking into account the compound error effect. To this end, we present a low-cost additional calibration circuit for the TDD multi-user (MU) massive MIMO system. Based upon this circuit design, we then propose a novel self-calibration scheme, namely inverse calibration, in the sense that this scheme relies on the inverse of the calibration coefficients. Simulation results show that the proposed inverse calibration outperforms the widely-used relative calibration, due to the fact that the former can offer a better compensation of the compound effect of both channel reciprocity error and channel estimation error.

\section{System Model}

We consider a TDD MU massive MIMO system as shown in Fig. 1, where we have $M$ antennas at the BS connected to individual RF chains, serving $K$ single-antenna UTs $(M \gg$ $K$ ) in the same time and frequency resources. We assume that the coherence time of the channel is larger than the time delay from the UL channel estimation to the DL transmission, such that the propagation channels on the UL and DL are equal.

As shown in Fig. 1, the UL and DL propagation channels are denoted by $\mathbf{H} \in \mathbb{C}^{M \times K}$ and $\mathbf{H}^{T}$ respectively, who have independent identically distributed (i.i.d.) entries, i.e. $C \mathcal{N}(0,1)$. Consider the same model of the BS RF frontends response as that in [8], the effective response matrices of the Rx and Tx RF frontends at the BS are represented by $M \times M$ diagonal matrices $\mathbf{H}_{b r}$ and $\mathbf{H}_{b t}$, whose $i$-th diagonal entries are $h_{b r, i}=A_{b r, i} \exp \left(j \varphi_{b r, i}\right)$ and $h_{b t, i}=A_{b t, i} \exp \left(j \varphi_{b t, i}\right)$, respectively, Here $A$ denotes amplitude, $\varphi$ denotes phase. As in [8], both amplitude and phase reciprocity errors are modelled as independent truncated Gaussian random variables. We assume that the statistical magnitudes of these truncated Gaussian distributed variables are static within the coherence time of the channel [11].

Consider the minimum mean-square error (MMSE) channel estimation for the UL training [4], [15], the MMSE estimate of the actual UL channel response $\mathbf{H}_{u}$ can be given by taking into account the effect of $\mathbf{H}_{b r}$ and $\mathbf{H}_{b t}$, as follows:

$$
\hat{\mathbf{H}}_{u}=\sqrt{1-\tau} \mathbf{H}_{b r} \mathbf{H}+\sqrt{\tau} \mathbf{N}_{u},
$$

where the estimation variance parameter $\tau \in[0,1]$ represents the estimation accuracy, i.e., $\tau=1$ represents the extreme case that there is not correlation between the estimation of $\mathbf{h}_{i}$ and its actual value, whereas $\tau=0$ corresponds to the perfect channel estimation without error [15]. In addition, the $M \times K$ noise matrix $\mathbf{N}_{u}$ has i.i.d. $C \mathcal{N}(0,1)$ elements, and $\rho_{u}$ denotes the expected UL transmit SNR. Then the BS uses the transpose of $\hat{\mathbf{H}}_{u}$ as the estimate of the DL channel $\hat{\mathbf{H}}_{d}$, i.e., $\hat{\mathbf{H}}_{d}=\hat{\mathbf{H}}_{u}^{T}$, whereas the actual effective DL channel is $\mathbf{H}_{d}=\mathbf{H}^{T} \mathbf{H}_{b t}$. By comparing $\hat{\mathbf{H}}_{d}$ and $\mathbf{H}_{d}$, we can rewrite the DL channel estimate $\hat{\mathbf{H}}_{d}$ as

$$
\hat{\mathbf{H}}_{d}=\sqrt{1-\tau} \mathbf{H}_{d} \mathbf{E}+\sqrt{\tau} \mathbf{N}_{u}^{T},
$$

where $\mathbf{E}=\mathbf{H}_{b t}^{-1} \mathbf{H}_{b r}$ denotes the channel reciprocity error. From (2), it can be seen that both additive and multiplicative distortions are introduced into the system due to the channel estimation and reciprocity errors, which causes the compound effect on the system performance. This in turn requires a careful design of calibration schemes to compensate for the impact caused by the reciprocity error, by taking the aforementioned compound effect into considerations.

Let a vector $\mathbf{s}=\left[s_{1} \cdots s_{k} \cdots s_{K}\right]^{T}$ denote the transmitted symbol to $K$ UTs, with the normalised symbol power per user. We assume that the symbols for different users are independent. Thus, $\mathbb{E}\left\{\mathbf{s s}^{H}\right\}=\mathbf{I}_{K}$. The BS calculates an $M \times K$ linear precoding matrix $\mathbf{W}$ to map the $K \times 1$ vector $\mathbf{s}$ into an $M \times 1$ transmit signal vector, $\mathbf{x}$, as given by

$$
\mathbf{x}=\sqrt{\rho_{d}} \lambda \mathbf{W s},
$$

where $\rho_{d}$ denotes the average transmit power at the BS. Note that the equal power allocation is considered in this work. $\lambda$ is a normalisation parameter to meet the transmission power constraint at the BS, i.e.,

$$
\mathbb{E}\left\{\|\mathbf{x}\|^{2}\right\}=\mathbb{E}\left\{\left\|\sqrt{\rho_{d}} \lambda \mathbf{W} \mathbf{s}\right\|^{2}\right\}=\rho_{d} .
$$

Hence, $\lambda$ can be calculated as $\lambda=\sqrt{\frac{1}{\mathbb{E}\left\{\operatorname{tr}\left(\mathbf{W W}^{H}\right)\right\}}}$. From (3), the collective received signal vector $\mathbf{y}$ at $K$ UTs is given by

$$
\mathbf{y}=\mathbf{H}_{d} \mathbf{x}+\mathbf{n}=\sqrt{\rho_{d}} \lambda \mathbf{H}^{T} \mathbf{H}_{b t} \mathbf{W} \mathbf{s}+\mathbf{n},
$$

where the DL received noise for all $K$ UTs is denoted by a $K \times 1$ vector $\mathbf{n}$, whose $k$-th element $n_{k} \sim C \mathcal{N}\left(0, \sigma_{k}^{2}\right)$. Assuming that $\sigma_{k}^{2}=1, \forall k$, then $\rho_{d}$ can be treated as the DL transmit SNR. For a given $k$-th UT, we have

$$
y_{k}=\sqrt{\rho_{d}} \lambda \mathbf{h}_{k}^{T} \mathbf{H}_{b t} \mathbf{w}_{k} s_{k}+\sum_{i=1, i \neq k}^{K} \sqrt{\rho_{d}} \lambda \mathbf{h}_{k}^{T} \mathbf{H}_{b t} \mathbf{w}_{i} s_{i}+n_{k} .
$$

Note that the received signal $y_{k}$ in (6) can be decomposed into three terms, where the first two terms accounts for the desired signal for the $k$-th UT and the inter-user interference among other $K-1$ UTs, respectively. Since $\mathbf{W}$ is calculated from the DL channel estimate instead of the actual DL 
channel response, the linear precoding schemes can be affected by both imperfect channel reciprocity and imperfect channel estimation. To address this issue, we introduce the calibration scheme as follows.

\section{SElf-CALIBRATION}

We introduce an $M \times M$ pre-precoding calibration matrix B to compensate for the imperfection of channel reciprocity [14], such that $\hat{\mathbf{H}}_{d}$ in (2) becomes

$$
\hat{\mathbf{H}}_{d, C L}=\sqrt{1-\tau} \mathbf{H}_{d} \mathbf{E B}+\sqrt{\tau} \mathbf{N}_{u}^{T} \mathbf{B} .
$$

The estimate of the DL channel response after applying calibration is denoted by $\hat{\mathbf{H}}_{d, C L}$, which is used to calculate the DL precoding matrix $\mathbf{W}$. According to [16], the minimum requirement to calibrate the $\mathrm{BS}$ antennas is that $\mathbf{E B}=c \mathbf{I}_{M}$, where the scalar $c \in \mathbb{C}_{\neq 0}$ can be unknown. The majority of the research on the reciprocity calibration proposes the design concept of calibration schemes based on this requirement [11], [13], [14].

The acquisition of the calibration matrix $\mathbf{B}$ contains two steps that are the estimation of $\mathbf{H}_{b t}, \mathbf{H}_{b r}$, and the calculation of $\mathbf{B}$ based on the estimates of $\mathbf{H}_{b t}$ and $\mathbf{H}_{b r}$. The widelyused relative calibration combines these two steps in a way that it estimates the ratio of $\mathbf{H}_{b t}$ to $\mathbf{H}_{b r}$, then obtains that $\mathbf{B}=c \mathbf{H}_{b t} \mathbf{H}_{b r}^{-1}$, e.g., [10], [11]. Besides the drawbacks of these techniques mentioned in Section I, these works ignore the impact of the channel estimation error on the calibration matrix, as discussed at the end of Section II. In this section, we introduce a novel self calibration scheme consisting of a) the estimation of $\mathbf{H}_{b t}$ and $\mathbf{H}_{b r}$ with simple additional circuits; b) a brand new design of the calibration matrix $\mathbf{B}$.

\section{A. RF Frontend Response Measurement}

Considering the calibration method for conventional MIMO systems in [12], we present a circuit design as illustrated in Fig. 1. To measure the effective response matrix of the BS RF frontend for the massive MIMO system, we have switching units attached to each antenna to set three modes: mode 1, the antenna connects to Tx or Rx RF frontend; mode 2, Tx and $\mathrm{Rx}$ RF frontends are connected; mode 3, no connection. We also have a reference signal source equally injected at each $\mathrm{Rx}$ Rf frontend by couplers. Then the measurement of $\mathbf{H}_{b t}$ and $\mathbf{H}_{b r}$ can be carried out in two steps which we call "self connection" and "half connection".

In the self connection, all switching units with the antennas are in mode 2 , and we disconnect the reference signal source. The baseband estimates the product of $h_{b t, i}$ and $h_{b r, i}$ by sending a pilot $p_{i}$ simultaneously, and receives $r_{i}=h_{b r, i} h_{b t, i} p_{i}+$ $u_{i}$, where the thermal noise $u_{i}$ is usually negligible due to the fact that the calibration SNR is sufficiently high, e.g., $20 \mathrm{~dB}$ in [12]. Hence, we have the estimate of $\mathbf{H}_{b t} \mathbf{H}_{b r}$, denoted by $\mathbf{R}_{\text {self }}$, as given by

$$
\mathbf{R}_{\text {self }}=\operatorname{diag}\left(r_{1} / p_{1}, \cdots, r_{i} / p_{i}, \cdots, r_{K} / p_{K}\right) .
$$

In the half connection, all switching units with the antennas are in mode 3 , and we have the reference signal source equally injected at all Rx RF frontends. Let an $M \times 1$ vector $\mathbf{p}_{\text {ref }}$ denote the reference signal vector. Then the collective received signal vector at the baseband is given by $\mathbf{r}_{h}=\mathbf{H}_{b r}\left(\mathbf{p}_{\text {ref }}+\mathbf{u}_{h}\right)$. Again, we ignore the effect of the measurement noise $\mathbf{u}_{h}$ with the assumption on the high calibration SNR. Hence, we have the estimate of $\mathbf{H}_{b r}$, denoted by $\mathbf{R}_{\text {half }}$, as given by

$$
\mathbf{R}_{\mathrm{HC}}=\operatorname{diag}\left(\mathbf{r}_{h}\right)\left(\operatorname{diag}\left(\mathbf{p}_{\text {ref }}\right)\right)^{-1} .
$$

As discussed in Section II, the reciprocity-error-related parameters are relatively static, i.e., the value of $\mathbf{H}_{b t}$ and $\mathbf{H}_{b r}$ changes in a much slower rate compared to the variations of the channel state. Hence, the acquired measurement of the BS RF responses, i.e., $\mathbf{R}_{\text {self }}$ and $\mathbf{R}_{\text {half }}$, can be applied within the the coherence time of the channel or even longer period [11].

\section{B. A New Design of the Calibration Matrix}

Based on (8) and (9), we can now calculate the calibration matrix B. In [12] the relative calibration scheme is considered, where the calibration matrix, denoted by $\mathbf{B}_{\mathrm{RC}}$, is given by $\mathbf{B}_{\mathrm{RC}}=\mathbf{R}_{\mathrm{SC}}\left(\mathbf{R}_{\mathrm{HC}}^{2}\right)^{-1}$. The widely-used relative calibration ignores the effect of the imperfect channel estimation, which can result in the estimation error amplification. In specific, we can see from (7) that using calibration matrix $\mathbf{B}_{\mathrm{RC}}$ may amplify the power of the estimation noise, or equivalently the channel estimation error, which additionally causes the enhancement of the inter-user interference, thus can even outweigh the benefit of calibration in certain cases, e.g., in the low region of $\rho_{u}$. We shall design a calibration matrix without the estimation error amplification.

Our design of the calibration matrix takes into account the aforementioned effect of the estimation error amplification. To this end, a desired calibration scheme should be able to compensate for the effect of the channel reciprocity error, as well as to reduce the noise power of the UL channel estimation, or equivalently reduce the estimation noise variance. Therefore, we devise a new calibration matrix, denoted by $\mathbf{B}_{\mathrm{IC}}$, which is given by

$$
\mathbf{B}_{\mathrm{IC}}=\mathbf{R}_{\mathrm{HC}}^{*}\left(\mathbf{R}_{\mathrm{HC}} \mathbf{R}_{\mathrm{SC}}^{*}\right)^{-1} .
$$

In the high calibration SNR regime, the calibration matrix $\mathbf{B}_{\mathrm{IC}}$ becomes the inverse of the product of $\mathbf{H}_{b t}^{*}$ and $\mathbf{H}_{b r}$. Thus we name the calibration scheme based on $\mathbf{B}_{\mathrm{IC}}$ as "Inverse Calibration". The performance of the traditional relative calibration and the proposed inverse calibration is compared in the following section.

\section{Simulation Results}

In this section, we show and discuss simulation results that compare the performance of the proposed inverse calibration (IC) and the traditional relative calibration (RC). Regarding the benchmark, we consider perfect channel reciprocity and no calibration (NC). Unless otherwise specified, we consider the statistical magnitudes of both amplitude and phase reciprocity errors as that defined in [8], [17], e.g., $\left(\alpha_{b t, 0}, \sigma_{b t}^{2},\left[a_{t}, b_{t}\right]\right)=\left(\alpha_{b r, 0}, \sigma_{b r}^{2},\left[a_{r}, b_{r}\right]\right)=$ $\left(0 \mathrm{~dB}, 1,\left[\begin{array}{lll}-4 & \mathrm{~dB}, 4 & \mathrm{~dB}\end{array}\right)\right.$, and $\left(\theta_{b t, 0}, \sigma_{\varphi_{t}}^{2},\left[\theta_{t, 1}, \theta_{t, 2}\right]\right)=$ 


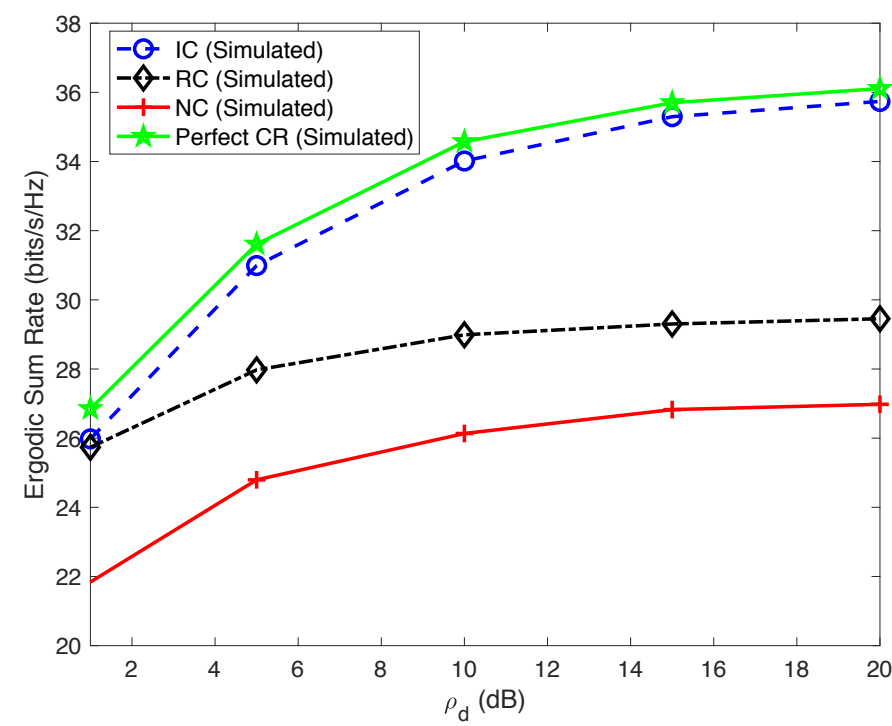

Fig. 2. Ergodic sum rate versus DL SNR in the presence of reciprocity error and channel estimation error.

$\left(\theta_{b r, 0}, \sigma_{\varphi_{r}}^{2},\left[\theta_{r, 1}, \theta_{r, 2}\right]\right)=\left(0^{\circ}, 1,\left[-50^{\circ}, 50^{\circ}\right]\right)$. And for the reference scenario with the perfect channel reciprocity (CR), we have $\sigma_{b t}^{2}=\sigma_{b t}^{2}=\sigma_{\varphi_{t}}^{2}=\sigma_{\varphi_{t}}^{2}=0$. The commonly considered MRT precoder is applied.

First, we investigate the ergodic sum rate of MRT with different calibration schemes. Let $M=100, K=10$, and reciprocity-error-related parameters as specified before, as well as $\tau=0.1$. From Fig. 2, we can observe that, for the MRT precoded system, the inverse calibration significantly outperforms the relative calibration, especially in the medium and high regions of $\mathrm{SNR}$, say above $1 \mathrm{~dB}$. We can also conclude that the proposed inverse calibration is more efficient than the relative calibration for MRT. To be specific, increasing the DL transmit power from $1 \mathrm{~dB}$ to $10 \mathrm{~dB}$, the performance of the inverse calibration increases by $31 \%$ (i.e., 8 bits $/ \mathrm{s} / \mathrm{Hz}$ ), while just less than $12 \%$ improvement (i.e., 3 bits $/ \mathrm{s} / \mathrm{Hz}$ ) can be achieved with the relative calibration. Fig. 2 indicates that both calibration schemes can work against the channel reciprocity error. However, the relative calibration suffers from a error ceiling factor, which can be caused by the channel estimation error amplification in the presence of the compound effect of non-reciprocity and imperfect channel estimation. This would eventually outweigh the benefit of applying the relative calibration. We shall take a closer look at this effect of estimation error amplification in the relative calibration.

In Fig. 3, we simply consider the output SINR (signalto-interference-plus-noise ratio) in $\mathrm{dB}$ as the performance metric. Similarly, we have $M=100, K=10$ and the MRT precoder. Let $\sigma_{A}^{2}=\sigma_{P}^{2}$ be the X-axis that captures the aggregated effect of both amplitude and phase reciprocity errors, i.e., $\left(\alpha_{b t, 0}, \sigma_{b t}^{2},\left[a_{t}, b_{t}\right]\right)=\left(\alpha_{b r, 0}, \sigma_{b r}^{2},\left[a_{r}, b_{r}\right]\right)=$

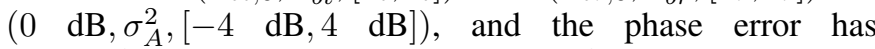
$\left(\theta_{b t, 0}, \sigma_{\varphi_{t}}^{2},\left[\theta_{t, 1}, \theta_{t, 2}\right]\right)=\left(\theta_{b r, 0}, \sigma_{\varphi_{r}}^{2},\left[\theta_{r, 1}, \theta_{r, 2}\right]\right)=$ $\left(0^{\circ}, \sigma_{P}^{2},\left[-50^{\circ}, 50^{\circ}\right]\right)$. We also consider the DL SNR

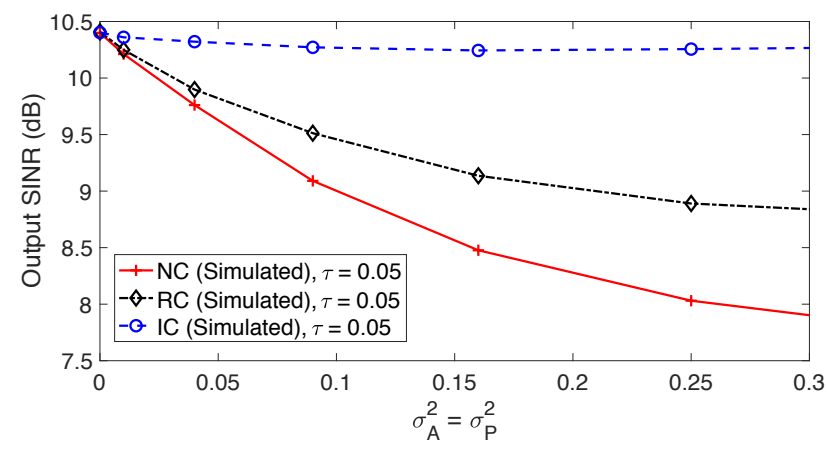

(a) $\tau=0.05$

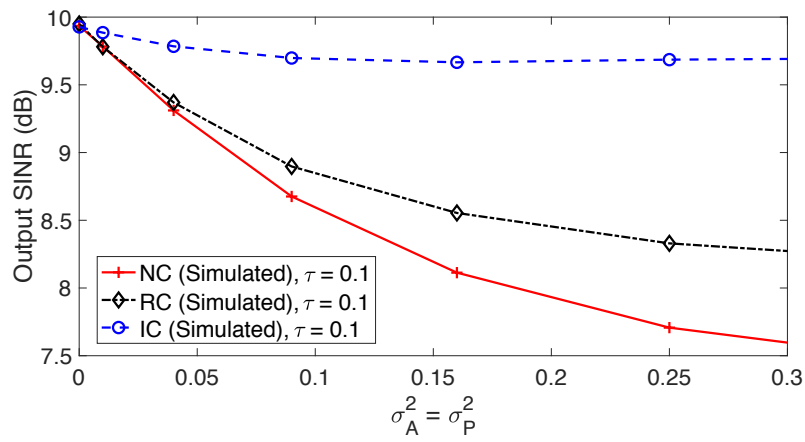

(b) $\tau=0.1$

Fig. 3. Output SINR versus reciprocity error variances with the different level of channel estimation error.

$\rho_{d}=10 \mathrm{~dB}$, as well as $\tau=0.05$ in Fig. 3(a) and $\tau=0.1$ in Fig. 3(b). It can be seen that due to its greater robustness to the compound effect of the reciprocity error and estimation error, the proposed inverse calibration still works correctly with only a minor performance degradation. whereas the gain of the relative calibration over no calibration vanishes in the case of the increased channel estimation error.

\section{CONCLUSION}

In this paper, we have proposed a novel self-calibration scheme, inverse calibration, for the TDD massive MU-MIMO system, by taking into account the compound effect of the multiplicative channel reciprocity error and the additive channel estimation error. A low cost calibration circuit based on the simple switch/coupler units has been presented, which allows the BS to perform either the inverse calibration or the traditional relative calibration. Considering the MRT precoder and the compound effect of both errors, We have shown that the inverse calibration in general outperforms the traditional relative calibration, thanks to the robustness of the inverse calibration to the compound error effect.

Further investigations including the theoretical analysis on both calibration schemes and the application on different precoding methods can be taken into account. In particular, analytical results of the ergodic sum rate for both schemes would provide valuable insights into the practical system design, including the guidance of the selection of different calibration schemes in physical data or control channels. 


\section{REFERENCES}

[1] E. G. Larsson, O. Edfors, F. Tufvesson, and T. L. Marzetta, "Massive MIMO for next generation wireless systems," IEEE Commun. Mag., vol. 52, no. 2, pp. 186-195, Feb. 2014.

[2] 3GPP TS 22.261 v15.3.0, "Service requirements for next generation new services and markets," Technical Specification, 122017.

[3] T. L. Marzetta, "Noncooperative cellular wireless with unlimited numbers of base station antennas," IEEE Trans. Wireless Commun., vol. 9, no. 11 , pp. 3590-3600, Nov. 2010.

[4] H. Yang and T. L. Marzetta, "Performance of conjugate and zeroforcing beamforming in large-scale antenna systems," IEEE J. Sel. Areas Commun., vol. 31, no. 2, pp. 172-179, Feb. 2013.

[5] J. Hoydis, S. ten Brink, and M. Debbah, "Massive MIMO in the UL/DL of cellular networks: How many antennas do we need?" IEEE J. Sel. Areas Commun., vol. 31, no. 2, pp. 160-171, Feb. 2013.

[6] L. Lu, G. Y. Li, A. L. Swindlehurst, A. Ashikhmin, and R. Zhang, "An overview of massive MIMO: Benefits and challenges," IEEE J. Sel. Topics Signal Process., vol. 8, no. 5, pp. 742-758, Oct. 2014.

[7] E. Björnson et al., "Massive MIMO systems with non-ideal hardware: Energy efficiency, estimation, and capacity limits," IEEE Trans. Inf. Theory, vol. 60, no. 11, pp. 7112-7139, Nov. 2014.

[8] D. Mi, M. Dianati, L. Zhang, S. Muhaidat, and R. Tafazolli, "Massive MIMO performance with imperfect channel reciprocity and channel estimation error," IEEE Trans. Commun., vol. 65, no. 9, pp. 3734-3749, Sept. 2017.

[9] M. Guillaud et al., "A practical method for wireless channel reciprocity exploitation through relative calibration," in Proc. 8th Int. Symp. on Signal Processing and Its Applications, vol. 1, Aug. 2005, pp. 403-406.

[10] H. Wei, D. Wang, H. Zhu, J. Wang, S. Sun, and X. You, "Mutual coupling calibration for multiuser massive MIMO systems," IEEE Trans. Wireless Commun., vol. 15, no. 1, pp. 606-619, Jan. 2016.

[11] C. Shepard et al., "Argos: Practical many-antenna base stations," in Proc. 18th Annu. Int. Conf. Mobile Comput. Netw., 2012, pp. 53-64.

[12] A. Bourdoux, B. Come, and N. Khaled, "Non-reciprocal transceivers in OFDM/SDMA systems: impact and mitigation," in Proc. IEEE Radio and Wireless Conf. (RAWCON), Aug. 2003, pp. 183-186.

[13] R. Rogalin et al., "Scalable synchronization and reciprocity calibration for distributed multiuser MIMO," IEEE Trans. Wireless Commun., vol. 13, no. 4, pp. 1815-1831, Apr. 2014.

[14] W. Zhang et al., "Large-scale antenna systems with UL/DL hardware mismatch: Achievable rates analysis and calibration," IEEE Trans. Commun., vol. 63, no. 4, pp. 1216-1229, Apr. 2015.

[15] D. Mi, D. Mehrdad, S. Muhaidat, and Y. Chen, "A novel antenna selection scheme for spatially correlated massive MIMO uplinks with imperfect channel estimation," in Proc. IEEE 81st Veh. Technol. Conf. (VTC), May 2015, pp. 1-6.

[16] T. Schenk, RF Imperfections in High-rate Wireless Systems: Impact and Digital Compensation. Springer Netherlands, 2008. [Online]. Available: https://books.google.co.uk/books?id=nLzk11P15IAC

[17] R1-100426, "Channel reciprocity modeling and performance evaluation," Alcatel-Lucent Shanghai Bell, Alcatel-Lucent, 3GPP TSG RAN WG1 \#59, 2010. 